\title{
Prevalence of subtypes of epithelial and sub-epithelial corneal dystrophy in Punjab-Pakistan
}

\author{
Shagufta Naz*, Nazia Ibrahim, Afia Iqbal, Saima Sharif, Farkhanda \\ Manzoor, Saba Fatima and Mehwish Iqtedar \\ Department of Zoology, Lahore College for Women University, Lahore-Pakistan \\ *Corresponding author's email: shagufta6@gmail.com \\ Citation \\ Shagufta Naz, Nazia Ibrahim, Afia Iqbal, Saima Sharif, Farkhanda Manzoor5, Saba Fatima and Mehwish \\ Iqtedar. Prevalence of subtypes of epithelial and sub-epithelial corneal dystrophy in Punjab-Pakistan. Pure and \\ Applied Biology. Vol. 7, Issue 2, pp872-881. http://dx.doi.org/10.19045/bspab.2018.700106
}

\begin{tabular}{llll}
\hline \hline Received: 07/03/2018 & Revised: 20/06/2018 & Accepted: 28/06/2018 & Online First: 29/06/2018 \\
\hline \hline
\end{tabular}

\section{Abstract}

This study was performed to determine the prevalence of subtypes of epithelial and subepithelial corneal dystrophy. The study was conducted between October, 2015 to June, 2016 on cases of epithelial and sub-epithelial during ophthalmological consultation at tertiary hospitals of Lahore where patients from different cities of Punjab including Okara, Sahiwal, Kasoor, Lahore, Gujranwala, Multan and northern areas also visited i.e., Mayo Hospital, General Hospital, Mughal Eye Hospital, Layton Rehmatullah Benevolent Trust (LRBT) Hospital, Ganga Ram Hospital, Al-Ehsan welfare Hospital and different hospitals of Gujranwala, Pakistan. The techniques used for the diagnosis of epithelial and sub-epithelial corneal dystrophy were visual acuity test by Snellen chart, Slit lamp biomicroscopy, Topography, Keratometer, and Pachymetry. In this study, 121 cases of epithelial and subepithelial corneal dystrophies were identified among which 60 were female and 61 were male patients. The main complaint was a drop in the visual acuity and loss of vision in all patients (100\%). The age group that was predominant in epithelial and sub-epithelial corneal dystrophy was 50-60 years and 80-90 years. Cases of epithelial corneal dystrophy were observed in all age groups but $37.09 \%$ cases were found in age 41-50 years. Epithelial, sub-epithelial and endothelial corneal dystrophy was more prevalent in males as compared to females in the patients of Punjab. This study will help us in genetic counseling of the affected individuals and their families to let them know the proper treatment of the disease.

Keywords: Biomicroscopy; Cornea; Keratometer; Visual acuity

\section{Introduction}

The human eye is a simple optical instrument that is designed to collect a major quantity of information about surroundings. It plays a most important role in vision. It is formed by only two positive lenses, cornea, and crystalline lens, that combine to project images of a scene onto the photoreceptor layer of the retina. The adult human eye resembles a sphere with a diameter of approximately $24 \mathrm{~mm}$ [1].

The cornea is a clear, crystalline, avascular structure comprising $16 \%$ of the ocular tunic. Structurally, the cornea is divided into five different layers including epithelium, Bowman's layer, stroma, Descemet membrane and endothelium [2]. Corneal dystrophies are bilateral, noninflammatory, progressive corneal diseases, characterized by deposition of non-native proteins in the different layers of the cornea [3]. Any variation in the normal corneal endothelium and Descemet layer leads to Epithelial and Sub-Epithelial corneal dystrophies. In many cases, a defective active fluid is produced by the corneal endothelium causes excessive edema of the corneal stroma. This condition 
effects on the clarity of the cornea and reduces visual acuity [4]. On the basis of histopathological and biomicroscopic features, corneal dystrophies are classified into 3 main groups; anterior, stromal and posterior corneal dystrophies $[4,5]$. The epithelium is the cornea's outermost region. It is filled with thousands of tiny nerve endings that make the cornea extremely sensitive to pain when rubbed or scratched [6]. Epithelial dystrophies usually manifest intraepithelial cysts and abnormal basement membrane.

The symptoms of epithelial and subepithelial corneal dystrophy are asymptomatic or recurrent erosions with pain, lacrimation, and blurred vision. Except for the bleb pattern, on-axis lesions may also cause blurred vision due to irregular astigmatism. The subtypes of epithelial and sub-epithelial corneal dystrophy are epithelial basement membrane dystrophy (EBMD), Epithelial recurrent erosion dystrophy (ERED), Subepithelial mucinous corneal dystrophy (SMCD), Meesmann corneal dystrophy (MECD), Lisch epithelial corneal dystrophy (LECD) and Gelatinous droplike corneal dystrophy (GDLD) [7].

\section{Materials and methods}

The diagnosis of epithelial and endothelial corneal dystrophies was performed on the basis of some methods like the visual acuity test was used to determine the smallest letters a person can read on a standardized chart (Snellen chart) held 20 feet away. Snellen's chart is used most frequently for testing visual acuity in which results are recorded as a fraction, Top number equates to the distance at which the test chart was presented and bottom number identifies the position on the chart of the smallest line read by the 'patient'.

Another method of corneal Pachymetry is the process of measuring the thickness of the cornea. It is used to perform corneal Pachymetry prior to surgery, for Keratoconus screening. By using the corneal Pachymetry the surgeon will reduce the chances of perforation of the eye and improves surgical outcome.

One more method that is corneal topography is a computer-assisted diagnostic tool that converts surface curvature of the cornea into three dimension map. The greatest advantage of corneal topography is its ability to detect irregular conditions invisible to most conventional testing. The threedimensional map is, therefore, a valuable aid to the diagnosis and treatment of a number of conditions such as in planning intraocular lens (IOL) implantation, refractive surgery and evaluating its results. A Keratometer also called ophthalmometer which is used to measure the curvature of the epithelial surface of the cornea, particularly for assessing the level and axis of astigmatism. It is also helpful for detecting irregular astigmatism, which is visible as distortion or regularity in the appearance of the cornea.

The last method that is used is orbscan, a valuable tool used pre-operatively to determine candidacy of patients. It calculates the elevation on the anterior and posterior surface of the cornea and helps in the measurement of corneal thickness and anterior chamber depth. After calculating results it will not only lead to evaluate whether the candidate is capable of laser refractive surgery but also show irregularities in the cornea that are difficult to detect by other means.

\section{Results}

Out of 121 cases of anterior corneal dystrophy that were analyzed, 61 cases $(50.41 \%)$ were of males and 60 cases $(49.58 \%)$ were of females. The age groups 0-10, 11-20, 21-30 and 31-40yeras had no case. But 10 cases $(8.26 \%)$ were observed in the age group 41-50. In this age group, 2 female $(3.33 \%)$ cases were observed and 8 male $(13.11 \%)$ cases were observed. There were 41 cases $(33.88 \%)$ observed in the age group of 51-60 and in this age group 27 were females (45\%) and 14 were males $(22.95 \%)$. In the age group of 61-70, almost 21 cases $(17.35 \%)$ were observed in which 
$12(20 \%)$ were females and $9(14.75 \%)$ were male. In the age group of 71-80, cases that were observed were $40(33.05 \%)$ in which $24(39.34 \%)$ were males and 16 $(26.66 \%)$ females and the last age group that was observed was 81-90 years. 9 cases $(7.43 \%)$ were observed in this age group in which $6(9.83 \%)$ were males and $3(5 \%)$ were females. Epithelial recurrent erosion dystrophy was found most prevalent in both genders $14(23.33 \%)$ cases of females and $11(18.03 \%)$ cases of males were observed and total cases of ERED were 25 (20.66\%). Subepithelial mucinous corneal dystrophy comprises only 17 cases $(14.04 \%)$ in which $8(13.33 \%)$ females and $9(14.75 \%)$ males were observed.

Snellen recordings of eyes of 121 patients were obtained. Six patients were found to have normal visual acuity (4.95\%). Moderate vision loss was recorded in eyes of $49(40.4 \%)$ patients. High vision loss was observed in the eyes of $76(62.8 \%)$ patients. Prevalence of visual acuity in 121 patients of epithelial and sub-epithelial corneal dystrophy explained in (Figure 1). Intraocular pressure in the patients of anterior corneal dystrophy was recorded. Out of 242 eyes of patients, 114 eyes $(47.10 \%)$ were with the normal IOP. 78 eyes of patients $(32.23 \%)$ were with the moderate-high IOP. 50 eyes $(20.66 \%)$ were with high IOP (Table 1).

From 121 cases of anterior corneal dystrophy that were analyzed, 21 (17.34\%) cases are of epithelial basement membrane dystrophy (EBMD) in which 10 (47.6\%) were males and $11(52.4 \%)$ were females. In males, the most representative age group was 71-80 years that had 13 (61.90\%) patients in which $5(50 \%)$ patients were male and $8(72.72 \%)$ were females. The other most representative age group was $81-90$ years that had 8 cases $(38.09 \%)$ of which 5 were $(50 \%)$ males and $3(27.27 \%)$ females were observed.

IOP in 21 patients of EBMD was recorded in which 10 males and 11 females were included. 9 patients $(42.85 \%)$ were with normal IOP in which 3 females and 6 males were present. 12 patients (57.14) were with moderate-high IOP that include 8 females and 4 males (Table 2)

Corneal thickness in the 21 patients of EBMD was recorded. 12 patients (57.14\%) were with a normal corneal thickness that is 500-550 $\mu \mathrm{m} .9$ patients $(42.85 \%)$ were with a less corneal thickness in which 6 males and 3 females were included. Corneal thickness decreases as age increase. Prevalence of visual disability in the patients of EBMD is described in (Figure 2).

prevalence of visual acuity in 121 patients

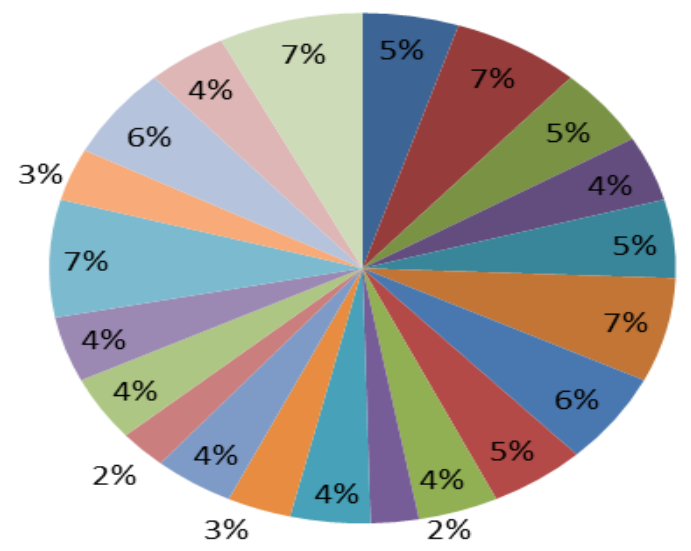

$$
\begin{gathered}
=6 / 6 \\
\square 6 / 18 \\
\square 6 / 12 \\
\square 6 / 20 \\
\square 6 / 21 \\
\square 6 / 22 \\
\square 6 / 23 \\
\square 6 / 24 \\
\square 6 / 25 \\
\square 6 / 29
\end{gathered}
$$

Figure 1. Prevalence of visual acuity in 121 patients of epithelial and subepithelial corneal dystrophy 
Table 1. IOP in the patients of anterior corneal dystrophy

\begin{tabular}{|c|c|c|}
\hline IOP & $\mathbf{N}$ & $\%$ \\
\hline 15 & 6 & 4.95 \\
\hline 16 & 8 & 6.61 \\
\hline 18 & 6 & 4.95 \\
\hline 19 & 5 & 4.13 \\
\hline 20 & 6 & 4.95 \\
\hline 17 & 8 & 6.61 \\
\hline 18 & 7 & 5.78 \\
\hline 17 & 6 & 4.95 \\
\hline 16 & 5 & 4.13 \\
\hline 20 & 3 & 2.47 \\
\hline 22 & 5 & 4.13 \\
\hline 25 & 4 & 3.30 \\
\hline 23 & 5 & 4.13 \\
\hline 21 & 3 & 2.47 \\
\hline 22 & 5 & 4.13 \\
\hline 21 & 5 & 4.13 \\
\hline 22 & 9 & 7.43 \\
\hline 26 & 4 & 3.30 \\
\hline 27 & 7 & 5.78 \\
\hline 29 & 5 & 4.13 \\
\hline 30 & 9 & 7.43 \\
\hline Total & 121 & 100.0 \\
\hline
\end{tabular}

The most representative age group epithelial recurrent erosion dystrophy (ERED) was 51-60 ages in which 7 (28\%) patients were included in which $5(35.71 \%)$ females and $2(18.18 \%)$ males were present. Another representative age group was $61-70$ that include 13 cases $(52 \%)$ in which $9(81.81 \%)$ males and $4(28.57 \%)$ females present. And the last representative age group was 71-80 that include 5 cases $(20 \%)$ in which $5(35.71 \%)$ females are included and no male was present.
IOP in 25 patients of ERED was recorded in which 11 males (44\%) and 14 females $(55 \%)$ were recorded. All patients $(100 \%)$ were with normal IOP (Table 3)

In 25 patients of ERED corneal thickness was recorded. 14 patients were with normal corneal thickness and 6 patients $(24 \%)$ were with moderate less corneal thickness. 5 patients $(20 \%)$ were with a less corneal thickness in which 2 males and 3 females were included. Prevalence of visual disability in the patients of ERED is illustrated in (Figure 3).

\section{Table 2. IOP in the patients of epithelial basement membrane dystrophy}

\begin{tabular}{|c|c|c|}
\hline IOP & $\mathbf{N}$ & $\mathbf{\%}$ \\
\hline 17 & 6 & 28.57 \\
\hline 20 & 3 & 14.28 \\
\hline 22 & 5 & 23.80 \\
\hline 25 & 4 & 19.04 \\
\hline 21 & 3 & 14.28 \\
\hline Total & 21 & 100.0 \\
\hline
\end{tabular}




\section{prevelence of visual acuity in the patients of EBMD}

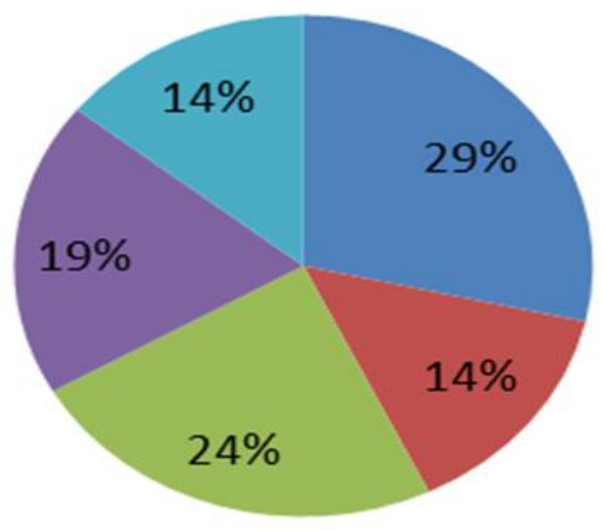

Figure 2. Prevalence of visual disability in the patients of EBMD

Table 3. IOP in the patients of ERED

\begin{tabular}{|c|c|c|}
\hline $\begin{array}{c}\text { IOP } \\
\text { (intraocular pressure) }\end{array}$ & $\begin{array}{c}\mathbf{N} \\
\text { (Total number) }\end{array}$ & $\begin{array}{c}\text { \% } \\
\text { (Percentage) }\end{array}$ \\
\hline 16 & 8 & 32 \\
\hline 18 & 6 & 24 \\
\hline 19 & 5 & 20 \\
\hline 20 & 6 & 24 \\
\hline Total & 25 & 100.0 \\
\hline
\end{tabular}

\section{prevalence of visual acuity in the patients of ERED}

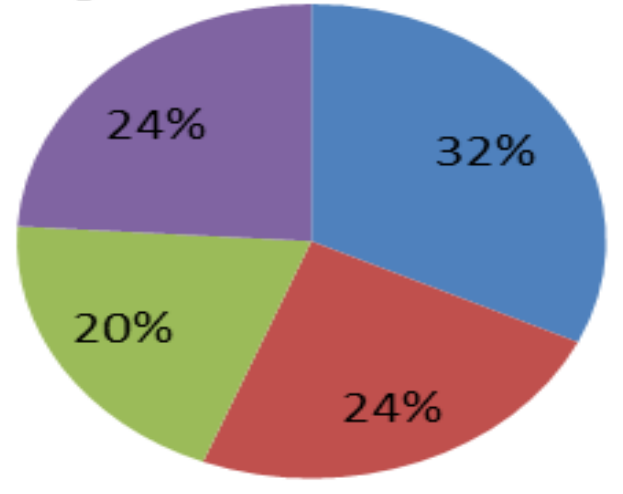

Figure 3. Prevalence of visual disability in the patients of ERED

Out of 121 cases of anterior corneal dystrophy, 17 cases (14.04\%) were of Subepithelial mucinous corneal dystrophy in which $9(52.94 \%)$ were males and 8
$(47.05 \%)$ were females. The most presentative age group was 41-50 that include 9 cases $(52.94 \%)$ in which 2 $(22.22 \%)$ females and $7(77.78 \%)$ males 
were present and another representative age group was 51-60 that include 8 cases $(47.05 \%)$ in which $6(75 \%)$ females and 2 $(25 \%)$ males were present.

IOP in the 17 patients of SMCD was recorded in which 9 males $(52.94 \%)$ and 8 females $(47.05 \%)$ were recorded. 13 patients have normal IOP in which 8 males and 5 females were present and 4 patients $(23.52 \%)$ were with high IOP in which 1 male and 3 females were present (Table 4) Corneal thickness in 17 patients of SMCD was recorded in which 9 females and 8 males were present. 4 patients $(23.52 \%)$ were with normal corneal thickness and 8 patients $(47.09 \%)$ were with a moderate less corneal thickness in which 5 males and 3 females were present. 5 patients $(29.41 \%)$ were with a less corneal thickness in which 3 males and 2 female were present. Prevalence of visual disability in patients of SMCD is explained in (Figure 4)

Out of 121 cases of anterior corneal dystrophy, 22 (18.19\%) cases were of Meesmann corneal dystrophy (MECD) in which $13(59.09 \%)$ patients were males and $9(40.9 \%)$ patients were females. The representative age group was 41-50 that includes 1 case of male (7.69\%). Another representative group was 51-60 that include 5 cases $(22.72 \%)$ in which $3(23.01 \%)$ males and 2 females (25\%) cases were present. Other representative age group was 61-70 that include 5 cases $(22.72 \%)$ in which 5 females $(62.5 \%)$ and no male present, the last representative age group was $71-80$ that include 11 cases $(50 \%)$ in which $10(76.9 \%)$ males and $1(12.5 \%)$ female was present.

IOP in 22 patients of MECD was recorded that included 13 males $(59.09 \%)$ and 9 females $(40.90 \%) .7$ patients $(33.3 \%)$ were with normal IOP and 15 patients $(68.18 \%)$ were with moderate-high IOP in which 8 males and 6 females were included. Prevalence of visual disability in the patients of MECD in (Figure 5).

Out of 121 cases of anterior corneal dystrophy, 18 (14.87\%) cases are of Lisch corneal dystrophy that includes $9(50 \%)$ males and $9(50 \%)$ females.

Representative age group was 51-60 that include 6 cases $(33.33 \%)$ in which 5 $(55.55 \%)$ females and 1 male $(11.11 \%)$ was present. Another representative group was 61-70 that includes 1 case of female and age group of 71-80 include 8 cases (44.44\%) in which $6(66.66 \%)$ males and $2(22.22 \%)$ females were present. Last representative group was 81-90 that include 2 (11.11\%) cases in which 1 male and 1 female were present.

IOP in 18 patients of LCD was recorded in which 9 females $(50 \%)$ and 9 males $(50 \%)$ were present. 9 patients were with moderate-high IOP but 9 patients were with high IOP in which 5 females and 4 males were present. Prevalence of visual disability in the patient of LECD by gender is illustrated in (Figure 6).

Out of 121 cases of anterior corneal dystrophy, $18(14.87 \%)$ cases are of gelatinous drop-like corneal dystrophy in which 9 were females and 9 were males. The representative age group 51-60 that include 16 cases $(88.89 \%)$ and the age group 71-80 include 2 cases (11.11\%) in which both males and females were included.

In 12 patients, IOP was recorded and all patients $(100 \%)$ were with high IOP. Out of 12 patients, 7 patients $(58.33 \%)$ were with less corneal thickness and 5 patients $(41.66 \%)$ were with normal corneal thickness. Prevalence of visual disability in the patients of GDLD is explained in (Figure 7).

\section{Statistical Analysis}

Data collected was arranged and chi-square test was performed for comparison between different age groups and was considered significant at $\mathrm{P}<0.05$. The variables examined were age, gender, intraocular pressure, corneal thickness and far visual acuity.

\section{Discussion}

The Ophthalmology Academy of America defined corneal dystrophies as "a group of inherited corneal diseases that are bilateral, 
slowly progressive, and symmetric" [8]. Endothelial and Sub-endothelial corneal dystrophies are a group of inherited disorders of the cornea that are caused by progressive accumulation of deposits within the cornea. These deposits are not caused by inflammation, infection, or trauma, but by genetic mutations that lead to abnormal proteins resulting in the accumulation of insoluble material within the cornea [9].

It is estimated that approximately 1.5 million children in the world are blind and corneal dystrophies are a major cause of blindness in these children. According to a recent study, $80 \%$ of world's blind people live in developing countries. In these countries, corneal diseases constitute $8 \%$ to $25 \%$ of cases of blindness which are responsible for $20 \%$ of childhood blindness. In the USA, the burden of corneal dystrophies is 897 per million [10]. In Europe, the prevalence is $13.9 \%$. Studies conducted in Japan and Australia revealed frequencies of corneal dystrophies $12.6 \%$ and $7 \%$ respectively [11].

In our study, we observed sixty-two $(n=62)$ cases of endothelial corneal dystrophies in between $<0-80$ years age group in which the most presented group was 41-50 years which included $37.09 \%$ patients. We observe different types of endothelial corneal dystrophies include; Fuchs corneal dystrophy, Posterior polymorphous corneal dystrophy, Congenital hereditary endothelial corneal dystrophy and X-linked endothelial dystrophy, but the most common form of dystrophy was congenital hereditary endothelial corneal dystrophy i.e., $32.25 \% \quad(n=20)$. The second most abundant dystrophic type was Fuchs corneal dystrophy which accounted for $25.8 \% \quad(n=16)$, then Posterior polymorphous corneal dystrophy and Xlinked endothelial dystrophy, both having $20 \%$ abundance $(\mathrm{n}=13)$.

An international classification of the corneal dystrophies has been developed that takes into account the chromosomal loci of the various corneal dystrophies as well as the responsible genes and their mutations. According to the French National Waiting List, dystrophies were the third most common indication for penetrating keratoplasty (PKP) and were divided into Fuchs dystrophy (65\%), lattice dystrophy (10\%), granular dystrophy (4\%), macular dystrophy (2\%), and other (20\%). A number of studies have been conducted throughout the world for the estimation of prevalence of corneal dystrophies. According to one of the study carried out in the USA, the prevalence of corneal dystrophies found is as 897 per million [12]. In a study conducted in Asian population states that endothelial dystrophies are more common in males, depicts dissimilarity in the presentation of endothelial dystrophies and this dissimilarity may be due to the difference in the races of both studies; the current study having Asians only. Therefore from these studies, we can deduce that the endothelial dystrophy is common in both Asians and Americans [12].

A study done in India found $9.6 \%$ prevalence of corneal dystrophies. Males were more commonly involved and stromal dystrophies were more prevalent in India [13]. While in our study male and female have an equal incidence of corneal dystrophies. An important parameter was also considered i.e., socioeconomic status was also observed in that study. Corneal dystrophies were more frequent in the high socioeconomic group (67\%) and less frequent in the low socioeconomic group (33\%). Like the current study, the Indian study also showed that stromal and posterior corneal dystrophies were more common, and anterior dystrophies were less common in India.

Genetic counseling provides information and support to people who have or may be at risk for, genetic disorders. This type of testing provides individuals and families with information, the nature of inheritance and implications of genetic disorder help them make informed medical and personal decisions [14]. 
Table 4. IOP in the patients of SMCD

\begin{tabular}{|c|c|c|}
\hline IOP & $\mathbf{N}$ & \% \\
\hline 17 & 8 & 47.05 \\
\hline 16 & 5 & 29.41 \\
\hline 26 & 4 & 23.52 \\
\hline Total & 17 & 100.0 \\
\hline
\end{tabular}

prevalence of visual disability in patients of SMCD

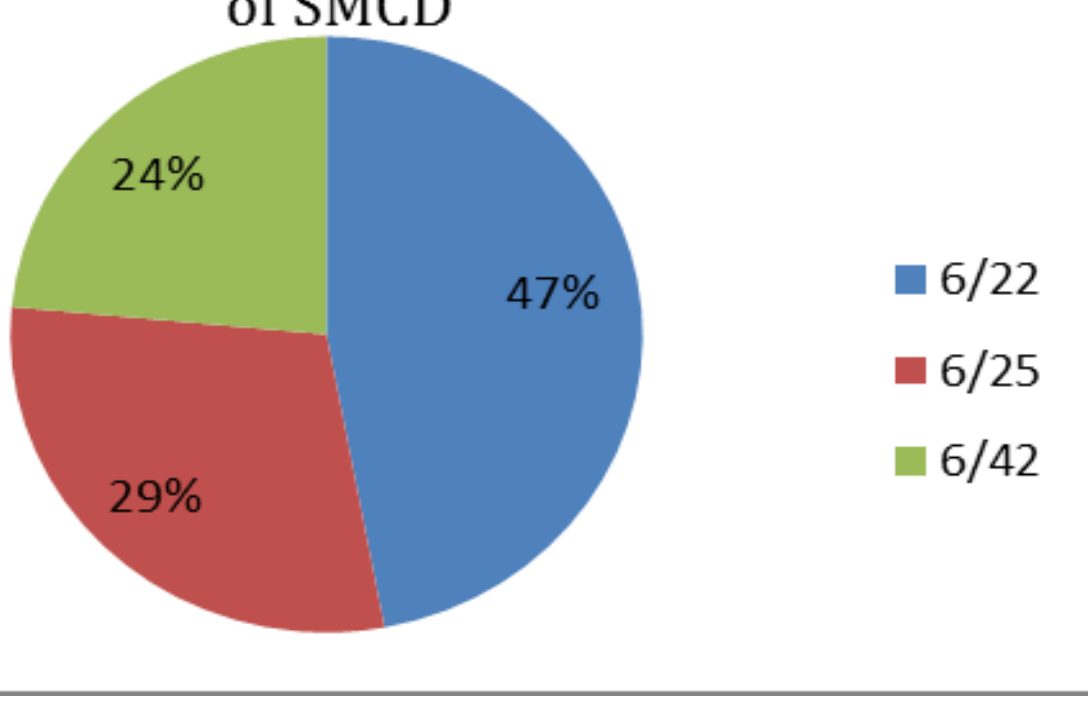

Figure 4. Prevalence of visual disability in patients of SMCD

\section{prevalance of visual disability in the patients of MECD}

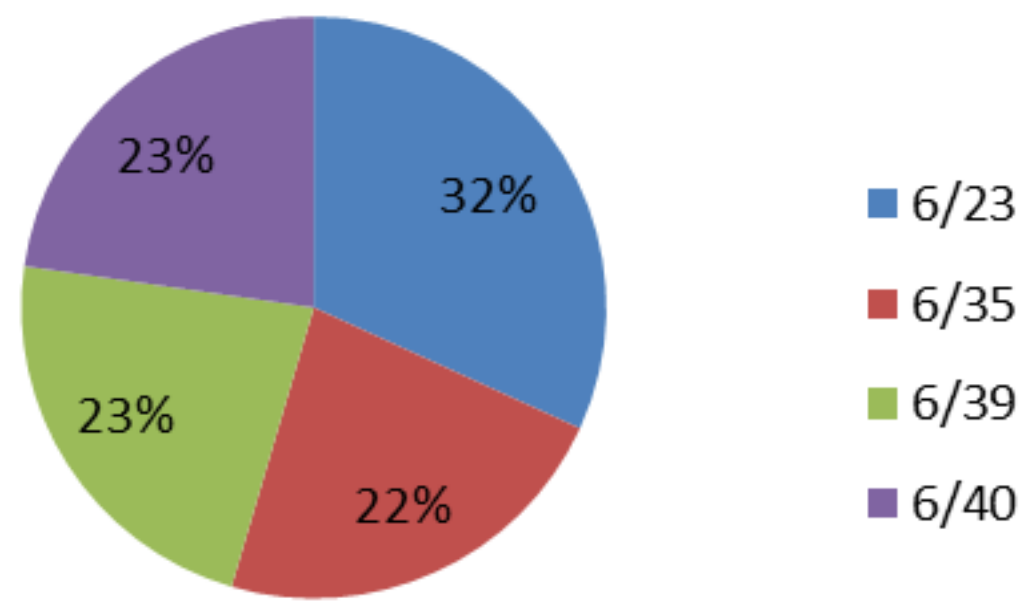

Figure 5. Prevalence of visual disability in the patients of MECD 


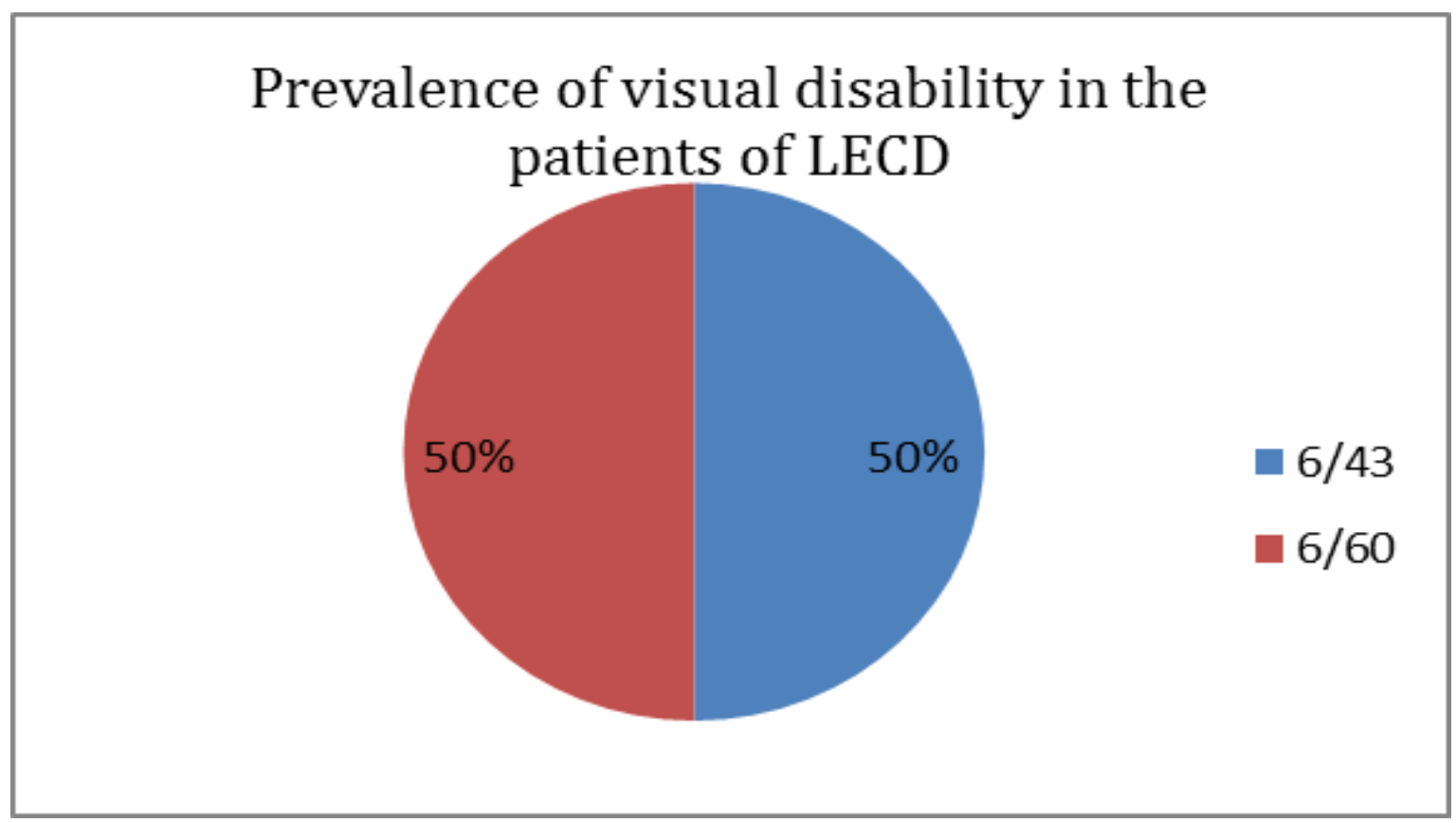

Figure 6. Prevalence of visual disability in the patient of LECD by gender

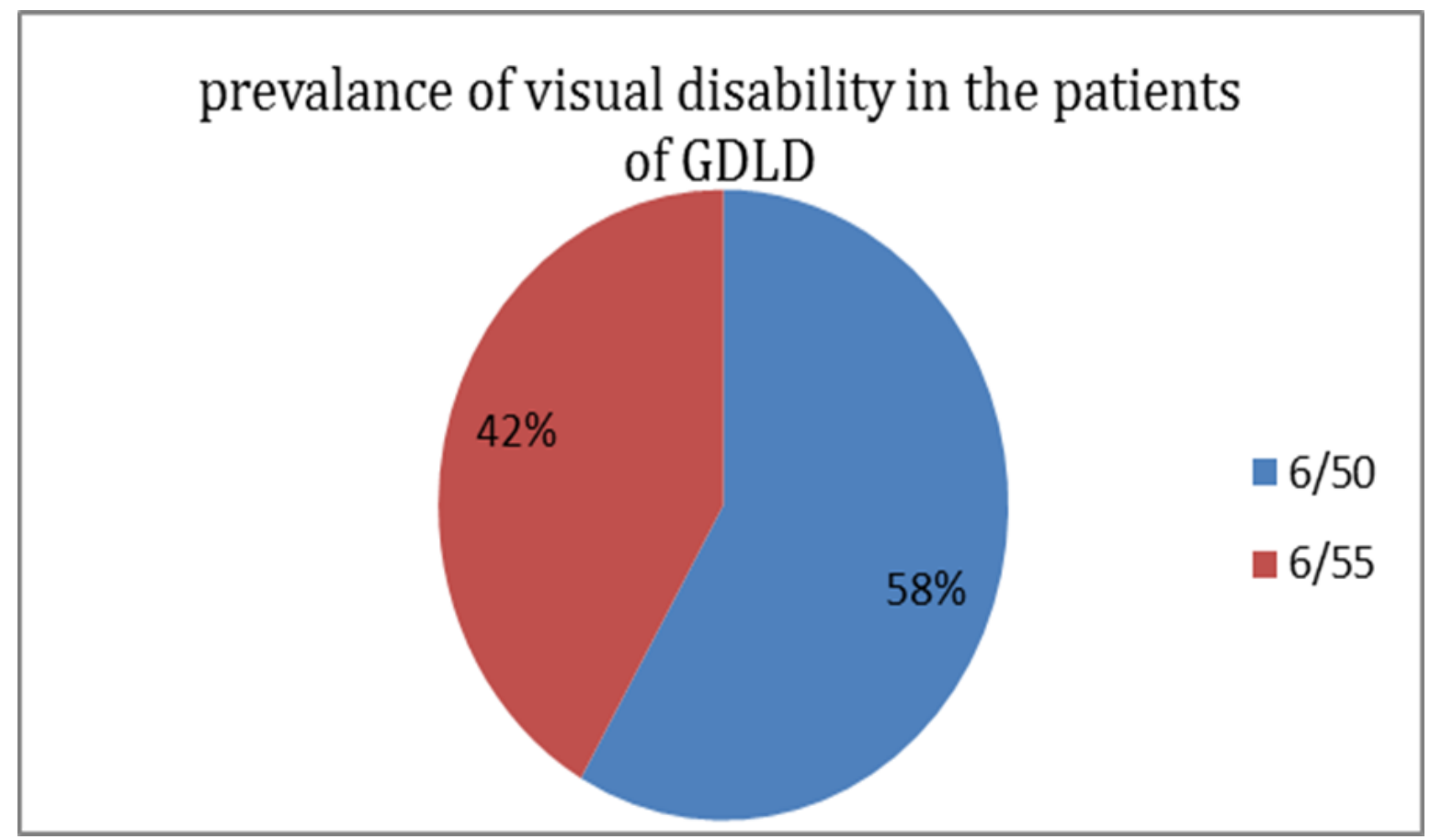

Figure 7. Prevalence of visual disability in the patients of GDLD

\section{Conclusion}

It can be concluded that epithelial and subepithelial corneal dystrophy is more prevalent in males as compared to females in the patients of northern areas of Pakistan. Epithelial and sub-epithelial corneal dystrophy is bilateral. The predominant age groups were 60-70 and 80-90. Vision impairment and vision loss due to epithelial and sub-epithelial corneal dystrophy were observed in $32.23 \%$ patients having moderate vision loss and $20.66 \%$ with high vision loss. 


\section{Authors' contributions}

Conceived and designed the experiments: $\mathrm{S}$ Naz, Performed the experiments: S Fatima, Analyzed the data: S Sharif, F Manzoor \& S Naz, Contributed materials/ analysis/ tools: S Fatima, M Iqtedar \& A Iqbal, Wrote the paper: S Naz \& N Ibrahim.

\section{Acknowledgments}

We sincerely thank Epithelial and Subepithelia corneal dystrophy patients and the staff of, General Hospital, Al-Ehsan welfare Hospital, Mughal eye and Mayo Hospital for their assistance in the clinical evaluation and collection of data.

\section{References}

1. DelMonte DW \& Kim T (2011). Anatomy and physiology of the cornea. $J$ Cataract Refract and Surger 37(3): 588-98.

2. Sohail AS, Brown SI \& Akiya S (2015). Prevalence of blindness in different countries. Am Aca Ophthalmol 12(7): 14-16.

3. Armitage WJ (2011). Preservation of human cornea. Trans Medicinal Hemotherapy 38: 143-7.

4. Klintworth GK (2009). Corneal dystrophies. Orphanet J Rare Dis 23: 4-7.

5. Godeiro KD, Coutinho AB, Pereira PR, Fernandes BF, Cassie A \& Burnier MN (2007). Histopathological diagnosis of corneal button specimens: an epidemiological study. Ophthalmic Epidemiol 14(2): 70-5.
6. Parthopratim D (2015). Outer most layer of cornea. Am Aca Ophthalmol 3(6): 12-15.

7. Klintworth A (2016). New classification of corneal dystrophy. Trans Am Ophthalmol Soc 107(7): 311-315.

8. Santo RM (2015). Prevalence of corneal dystrophy. Act Ophthalmol 30 (9): 11-16.

9. Weiss J, Moller HU, Lisch W, Kinoshita S, Aldave AJ \& Belin MW (2010). The IC3D classification of corneal dystrophies. Cornea 29(12): 1496.

10. Hergersberg W (2015).Prevalence of corneal dystrophy. Act Ophthalmol. 30(7): 12-17.

11. Polack FM, Bourne WM, Forstot SL \& Yamaguchi T (1980). Scanning electron microscopy of posterior polymorphous corneal dystrophy. Am J Ophthalmol 89 (4): 575-84.

12. Auw-Hädrich C \& Witschel H (2002). Corneal dystrophies in the light of modern molecular genetic research. Ophthalmologe 99: 418-426.

13. Sohail I, Noreen F, Nawaz S, Ahmed S, Zafar H \& Niaz M (2015). Frequency of corneal dystrophies on the basis of histopathology in surgically removed corneas. Pak J Med Ass 65(10): 1056-1059.

14. Zenteno AH, Cogan DG, Donaldson D \& Kawabata $\mathrm{T}$ (2012). International classification of corneal dystrophy. Am Aca Ophthalmol 4(9): 12-14. 